\title{
Automation technologies for strawberry harvesting and packing operations in Japan ${ }^{1}$
}

\author{
Shigehiko Hayashia,*, Satoshi Yamamoto ${ }^{a}$, Shogo Tsubota ${ }^{\mathrm{a}}$, Yoshiji Ochiai ${ }^{\mathrm{a}}$, Ken Kobayashi ${ }^{\mathrm{a}}$, \\ Junzo Kamata ${ }^{\mathrm{b}}$, Mitsutaka Kurita ${ }^{\mathrm{b}}$, Hiroyuki Inazumi ${ }^{\mathrm{b}}$ and Rajendra Peter ${ }^{\mathrm{b}}$ \\ ${ }^{a}$ BRAIN, National Agriculture and Food Organization, Nisshin, Kita, Saitama, Japan \\ ${ }^{\mathrm{b}}$ Shibuya Seiki Co. Ltd, Takaoka, Matsuyama, Japan
}

Submitted 29 October 2013; accepted 16 December 2013

\begin{abstract}
This paper describes a strawberry-harvesting robot, a packing robot, and a movable bench system. The harvesting and packing operations in strawberry production require harder, more time-consuming work compared to other operations such as transplanting and chemical spraying, making automation of these tasks desirable. Since harvesting and packing operation account for half of total working hours, automation of these tasks are strongly desired. First of all, based on the findings of many studies on strawberry-harvesting robots for soil culture and elevated substrate culture, our institute of the Bio-oriented Technology Research Advancement Institution and Shibuya Seiki developed a commercial model of a strawberry-harvesting robot, which is chiefly composed a cylindrical manipulator, machine vision, an end-effector, and traveling platform. The results showed an average $54.9 \%$ harvesting success rate, $8.6 \mathrm{~s}$ cycle time of picking operation, and $102.5 \mathrm{~m} / \mathrm{h}$ work efficiency in hanging-type growing beds in an experimental greenhouse. Secondly, a prototype automatic packing robot consisting of a supply unit and a packing unit was developed. The supply unit picks up strawberries from a harvesting container, and the packing unit sucks each fruit from calyx side and locates its orientation into a tray. Performance testing showed that automatic packing had a task success rate of $97.3 \%$, with a process time per fruit of $7.3 \mathrm{~s}$. Thirdly, a movable bench system was developed, which makes planting beds rotate in longitudinal and lateral ways. This system brought high density production and labour saving operation at a fixed position, such as crop maintenance and harvesting. By setting up the main body of a strawberry-harvesting robot on working space, unmanned operation technique was developed and tested in an experimental greenhouse. Field experiments of these new automation technologies were conducted and gave a potential of practical use.
\end{abstract}

Keywords: Strawberry harvesting, packing operations, automation technologies

\section{Introduction}

The strawberry (Fragaria $\times$ ananassa Duch.) has gained worldwide popularity but is one of the most difficult crops to handle because of its soft pericarp. In Japan, 190,700 $t$ were produced in 2008 from a cultivated area of 6471 ha [1], mostly for fresh consumption. In forcing culture, strawberry plants are transplanted into greenhouses in September and harvested from November until April of the following year [2]. The fruits are harvested early in the morning, before the temperature of the fruit rises, which causes them to become soft. The workers also need to select the mature red fruits from among the many fruits that have set. These factors result in long working hours during the harvesting period. To harden the pericarp, the harvested fruits are precooled in storage before packing. They are sorted using

\footnotetext{
${ }^{1}$ Paper presented at 2nd International Strawberry Congress, Hoogstraten, Belgium, Sept 4-6, 2013.

*Corresponding author: Shigehiko Hayashi, BRAIN, National Agriculture and Food Organization, 1-40-2, Nisshin, Kita, Saitama, Japan. E-mail: shigey@affrc.go.jp.
} 
a grading standard (size and shape) that differs according to the production area. The strawberries are methodically placed in a single-layer soft tray or in plastic boxes in two layers to protect them from vibration during transport to the market and to make them visually appealing. Nearly all operations, including harvesting, sorting, and packing, are performed manually; therefore, automating the harvesting and packing operations in strawberry production is highly desirable.

Researchers have overcome many challenges to automate fruit harvesting operations for strawberries as well as for other fruits, such as oranges [3-5], apples [6], tomatoes [7], cherry-tomatoes [8], eggplants [9], cucumbers [10, $11]$, and so on. Studies on strawberry-harvesting robots for soil culture and an elevated substrate culture were started in Japanese universities [12-14], while the Bio-oriented Technology Research Advancement Institution (BRAIN) and Shibuya Seiki created a prototype commercial model robot. Moreover, many types of fruits have been graded and packed by robots in packing facilities [15-17]. Several grading systems for strawberries have also been designed [18-20]; however, strawberries are too delicate to be handled by mechanical hands. To automate this packing task, our institute developed a prototype packing robot that suctions strawberry fruit from the calyx side using machine vision.

This paper describes a strawberry-harvesting robot, which moves around a greenhouse, and a picking robot, which uses a machine vision technique. Furthermore, the combined use of a harvesting robot and a movable bench system in strawberry production is proposed.

\section{Strawberry-harvesting robot}

\subsection{System components}

A strawberry-harvesting robot was developed based on the findings obtained with our previous prototype [21]. The harvesting robot consists of a cylindrical manipulator, an end-effector, a machine vision unit, and a tray storage unit. Figure 1 shows a photograph of the robot.

A three-degree-of-freedom (DOF) cylindrical manipulator was set up as part of an inverted structure in which the lower part of the manipulator was positioned upward to ensure there was space under the end-effector. The operational angles for rotation and the lengths in the vertical and horizontal directions are $250^{\circ}, 400 \mathrm{~mm}$, and $300 \mathrm{~mm}$, respectively. The end-effector consists of a gripper with two fingers for cutting the peduncle and a reflection-type photoelectric sensor for confirming the presence of the picked fruit (Fig. 2). An interchangeable blade and a stopper are attached

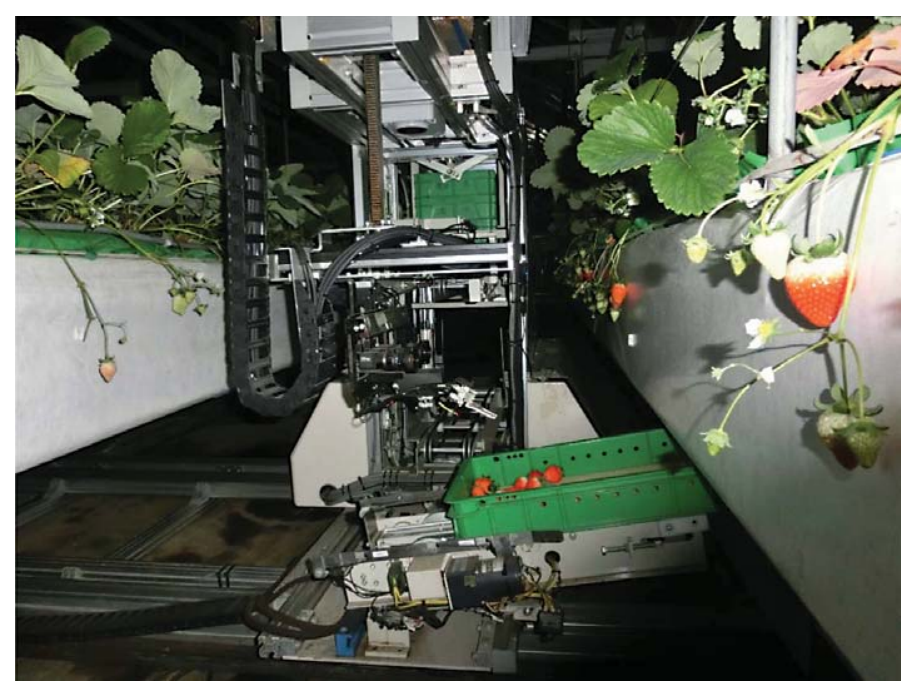

Fig. 1. Strawberry-harvesting robot. 


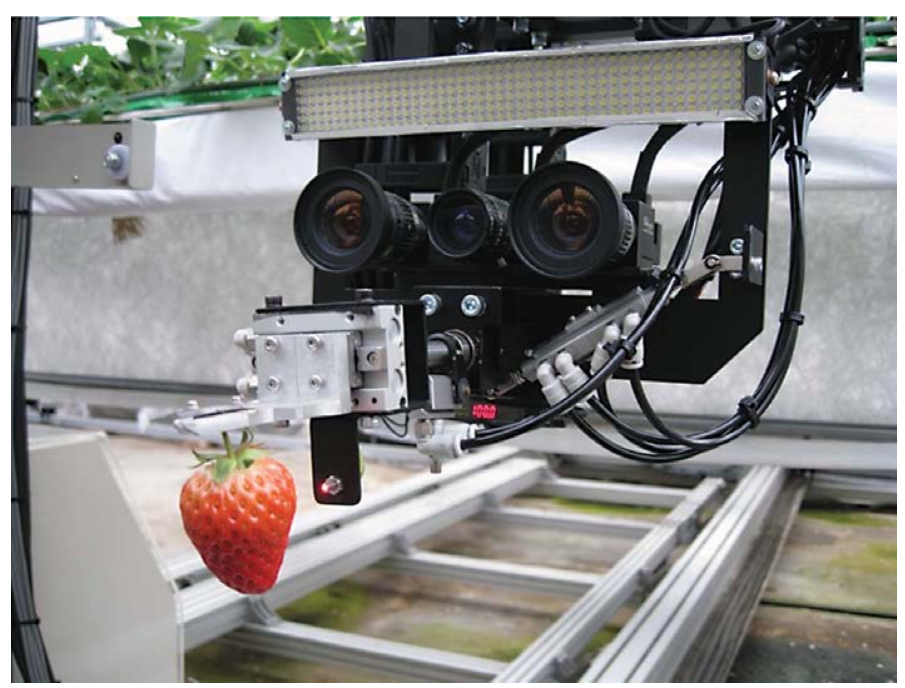

Fig. 2. Picking a strawberry with the end-effector.

to the finger. Cushioning material is pasted to the contact side of the finger to enable it to simultaneously hold and cut the peduncle. The machine vision unit has a rectangular light-emitting diode (LED) source and three colour charge-coupled device cameras. The two side cameras are used to provide stereo vision to determine the position in the 3D space and maturity level of each fruit. The camera located in the centre is used to detect the peduncle and calculate its inclination. The tray storage is separated into two layers and is configured to accommodate four trays. Each tray accommodates 84 fruits, resulting in a total of 336 fruits.

\subsection{Movement in the experimental greenhouse}

We developed the travelling platform to allow the harvesting robot to move around the greenhouse [22]. The sideways table on which the harvesting robot is mounted moves in the traversing direction, starting from the home position, and stops at the entry point of a path. Four empty trays are loaded onto the harvesting robot. The harvesting robot then moves along the path and picks the fruits on the right-hand side in accordance with the picking procedure, before reaching the end of the path. On the way back, the harvesting robot picks the fruits on the left-hand side. When the picking task for the path is finished, the harvesting robot discharges the filled trays to the tray stocker and returns to its home position.

The picking task is performed after the travelling platform has halted. The machine vision detects red fruits and assesses their maturity level. If the fruit's maturity level has reached the selected threshold level, the machine vision calculates its position in the 3D space. The end-effector then moves to a position about $275 \mathrm{~mm}$ in front of the target fruit, and the centre camera detects the peduncle to be cut with the fingers. The picked fruit is placed in the tray.

\subsection{Harvesting performance in the field}

The successful harvesting rate (SHR) was measured to assess the basic performance of the strawberry-harvesting robot. A test area of $35.25 \mathrm{~m}$ at both sides of one pathway was set. The fruits with a maturity of $80 \%$ or more were marked as the target fruits and counted before the robot was operated. The harvesting robot then executed automatic harvesting with the fixed parameters of an $80 \%$ maturity level and the two-try mode.

The harvesting robot was successfully able to pick 73 out of 133 mature fruits, giving an SHR of 54.9\%. This value of 73 included three fruits picked successfully on the second attempt. Of the 60 fruits that failed to be picked, 57 fruits were not detected as target fruits because they were eclipsed by other fruits. The other three fruits failed to be cut by the end-effector owing to errors in peduncle detection. 


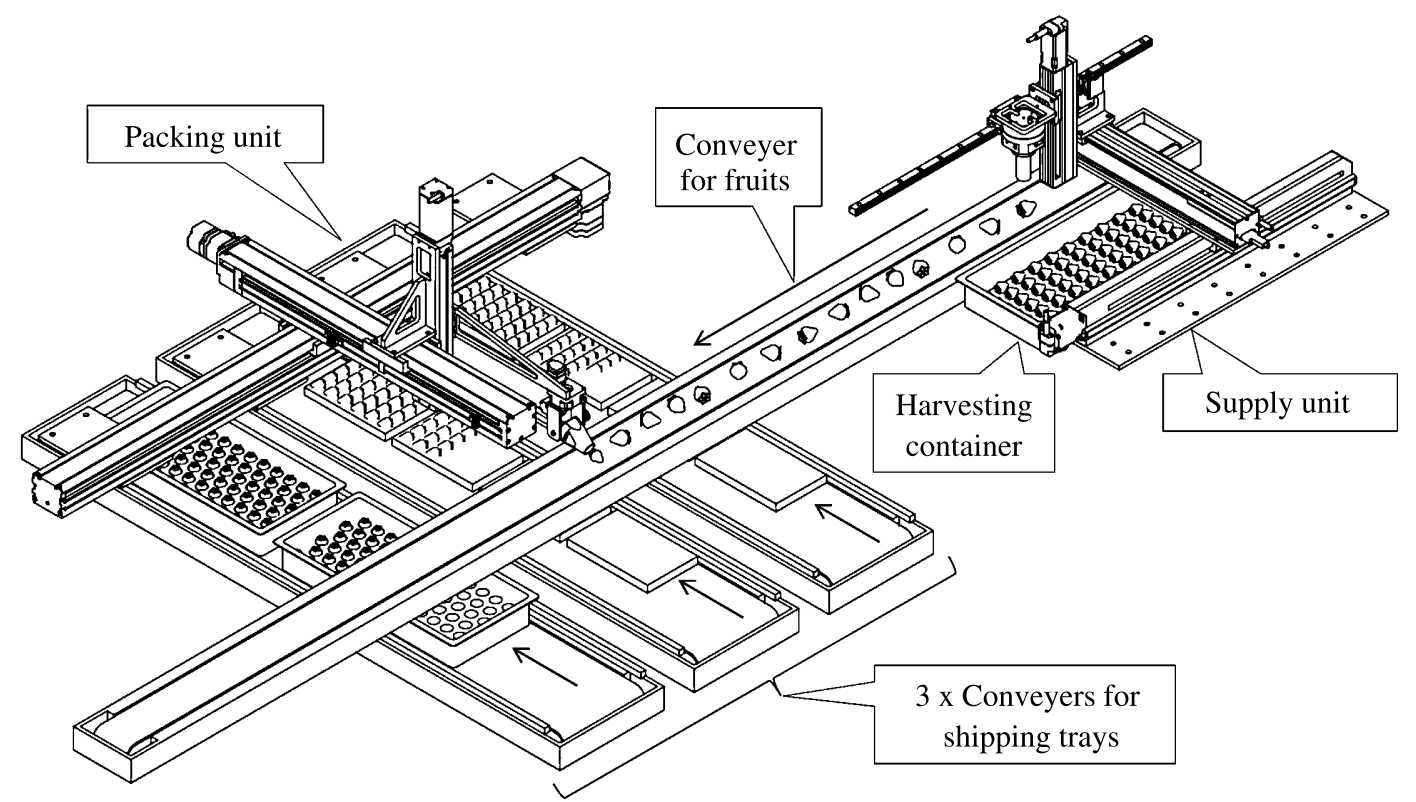

Fig. 3. Schematic diagram of automatic packing robot for strawberries [23].

\section{Strawberry-packing robot}

\subsection{System components}

We developed a prototype automatic packing robot [23] consisting of a packing unit and a supply unit (Fig. 3). The supply unit picks up strawberries from the harvesting container and places them on a conveyer that transports them to the packing unit. The packing unit grades each fruit according to its size and locates its orientation and position using a machine vision system. Using this geometric information, the packing unit picks up the fruit and methodically places it in a shipping tray. Although these two units are controlled by one PC, they each operate independently. Only the fruit conveyer, which conveys fruits from the supply unit to the packing unit, is affected by the motion status of both units; the conveyer stops when the supply unit places the fruit on it or when the packing unit picks up the transported fruit.

\subsection{Supply unit}

The supply unit, consisting of the machine vision system, a suction hand, a manipulator with three DOFs, and a fruit conveyer for moving the fruits to the packing unit, picks up the fruits at random positions from the harvesting container $(\mathrm{L} 26 \mathrm{~cm} \times \mathrm{W} 54 \mathrm{~cm} \times \mathrm{H} 8 \mathrm{~cm}$ ) and then places them on the fruit conveyer. A photoelectric sensor is installed at a height of $15 \mathrm{~mm}$ above the conveyer around the placement point of a fruit, and the supply unit stops the operation when the photoelectric sensor detects a fruit. After the supply unit places the fruit on the conveyer, it is detected by the photoelectric sensor, and the manipulator decelerates and stops. The suction hand always releases the fruit at the same height of less than $10 \mathrm{~mm}$ above the conveyer, regardless of its size or position.

\subsection{Packing unit}

We have added new functions to the current strawberry pickup equipment [25] such as the packing unit of the automatic packing robot. The packing unit's suction device is equipped with a step motor, which tilts the suction tube between $-90^{\circ}$ and $+90^{\circ}$. When the suction device picks up the fruit, it tilts the suction tube $10^{\circ}$ away from the 


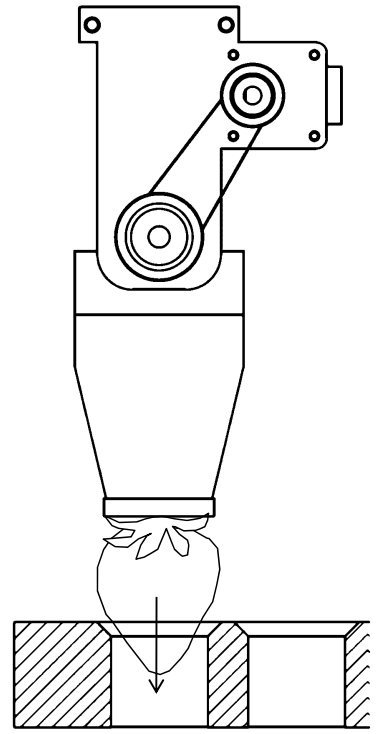

(a) Motion of placing a fruit in a returnable tray.

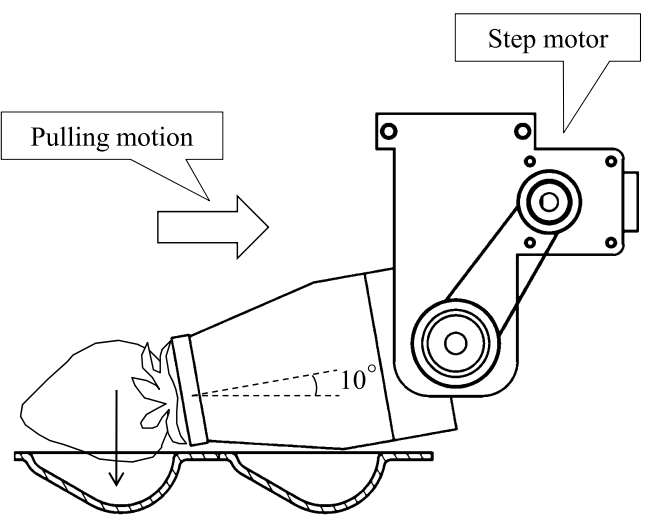

(b) Motion of placing a fruit in a single-layer tray.

Fig. 4. Tilt motion of the packing unit's suction device [23].

horizontal, and the fruit is packed vertically into the returnable tray, as shown in Fig. 4. The tilt angle of the suction tube is set at $10^{\circ}$ again when the fruit is packed in the single-layer tray (Fig. 4). We added a 30 mm pulling motion of the suction device after the placing motion in the longitudinal direction from the hollow of the single-layer tray and confirmed that the fruits were released smoothly.

\subsection{Performance testing}

The performance testing of the automatic packing robot was carried out from December 2010 to February 2011 in Saga Prefecture, one of Japan's main strawberry-producing areas. Fruits of the cultivar 'Sagahonoka' were sorted by a farmer into three grades (small, medium, and large) and were tested separately by grade.

The task success rate of the complete automatic packing robot was $97.3 \%$. The task success rates of the supply unit and the packing unit individually were $98.0 \%$ and $99.3 \%$, respectively. In this test, the supply unit picked up all of the fruits from a harvesting container and safely placed them on the conveyer. However, the spacing between fruits was sometimes too short, and the packing unit detected a collision risk. On another occasion, the packing unit failed to pick up a large fruit and did not retry. The overall process time per fruit was $7.3 \mathrm{~s}$ owing to the parallel operation of two units, whereas the sorting and packing times by hand ranges from approximately 5 to $8 \mathrm{~s}$ per fruit. Considering the feasibility of the automatic packing system, speeding of the overall process time would be necessary in the further study.

\section{Movable bench system integrated with robot harvesting technique for strawberries}

\subsection{System components}

A new movable bench system, measuring $4.3 \mathrm{~m}$ long and $9.4 \mathrm{~m}$ wide, was developed based on the previous prototype [24]. It chiefly comprises two longitudinal conveying units, two lateral conveying units, two nutrient supply units, a chemical sprayer, 16 planting benches, and a control unit, as shown in Fig. 5. The planting bench is composed of a frame, three planters with a length of $1.2 \mathrm{~m}$, a gutter, and four rollers, and it moves on a set of rails $1.7 \mathrm{~m}$ apart. The 


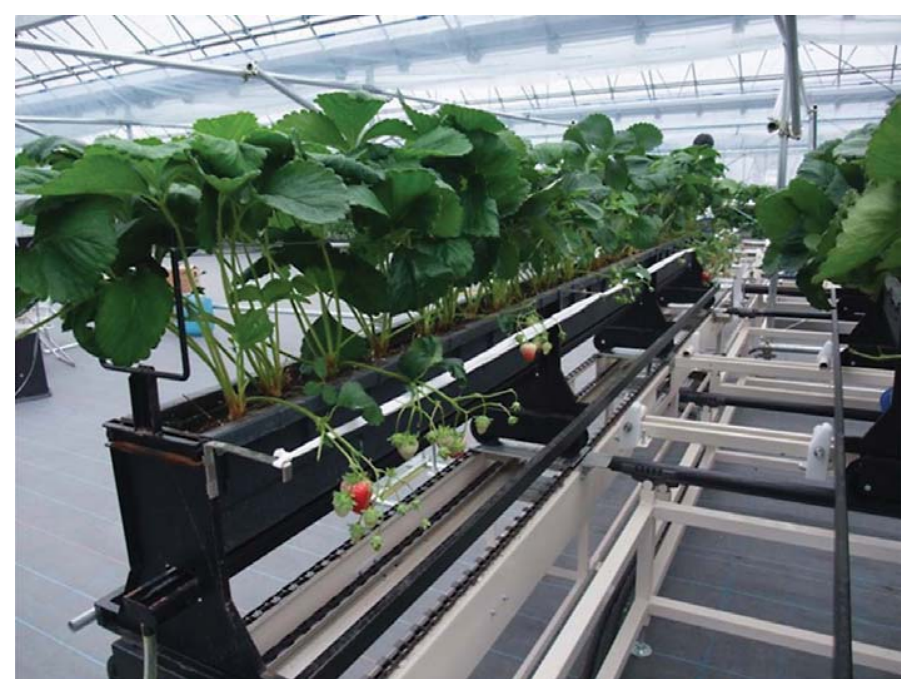

Fig. 5. Movable bench system for strawberries.
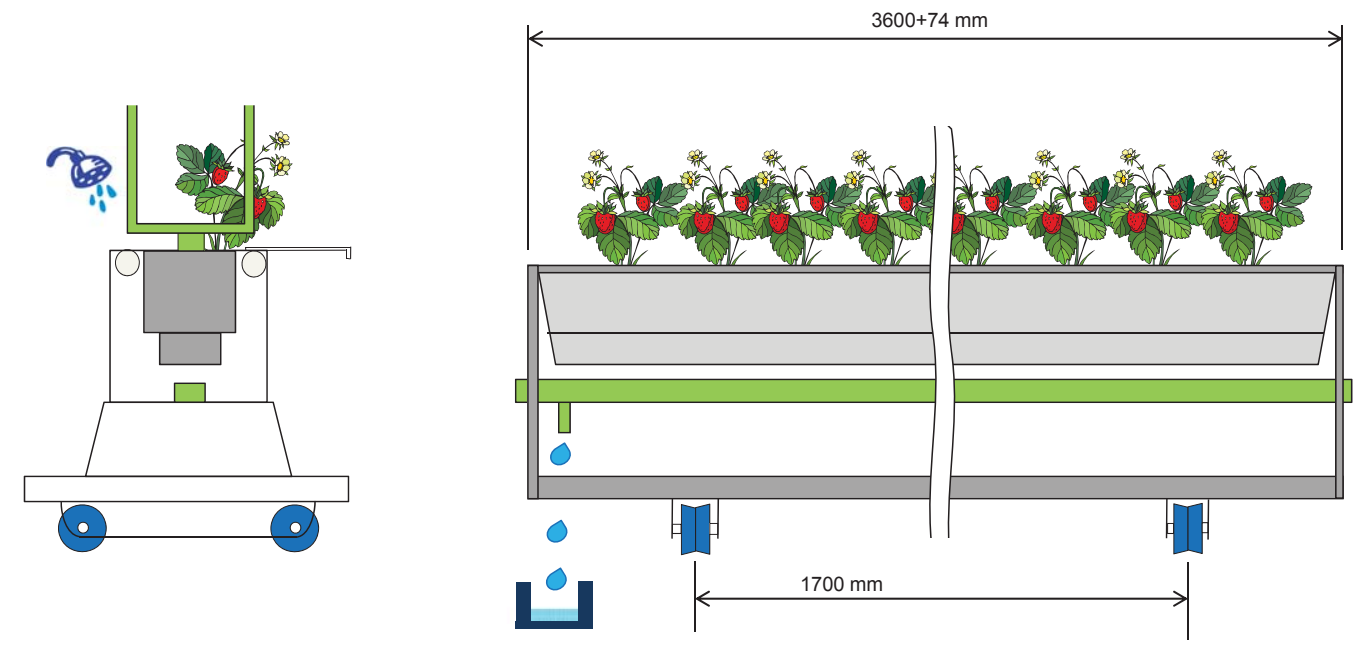

Fig. 6. Schematic diameter of planting bench for movable bench system.

bench is $3.67 \mathrm{~m}$ long, $0.41 \mathrm{~m}$ wide, and $0.46 \mathrm{~m}$ high, as shown in Fig. 6 . The planting benches are conveyed $500 \mathrm{~mm}$ at a time, because the pull-in rods have ratchets at $500 \mathrm{~mm}$ intervals. The system's two longitudinal conveying units are composed of two $400 \mathrm{~W}$ waterproof electric motors, lever-crank links, and pull-in rods for the benches. The two lateral conveying units are composed of a stainless-steel chain, a sprocket, and a $200 \mathrm{~W}$ waterproof electric motor. The lateral conveying units are designed to convey the planting bench to the other side of the longitudinal conveying unit, and they have three speed modes: high, medium, and low, which can be selected to suit the task, for instance, transplanting, crop management, harvesting, or chemical spraying.

Because of the way each bench circulates, the nutrient solution is supplied by a water nozzle installed in the middle of both lateral conveying units. Watering begins when the planting bench starts moving. The amount of nutrients supplied is controlled via a valve in the water nozzle, the speed of lateral conveying, and the number of waterings needed to meet the daily water requirement of strawberry plants (generally $300-400 \mathrm{~mL}$ per day). The chemical 


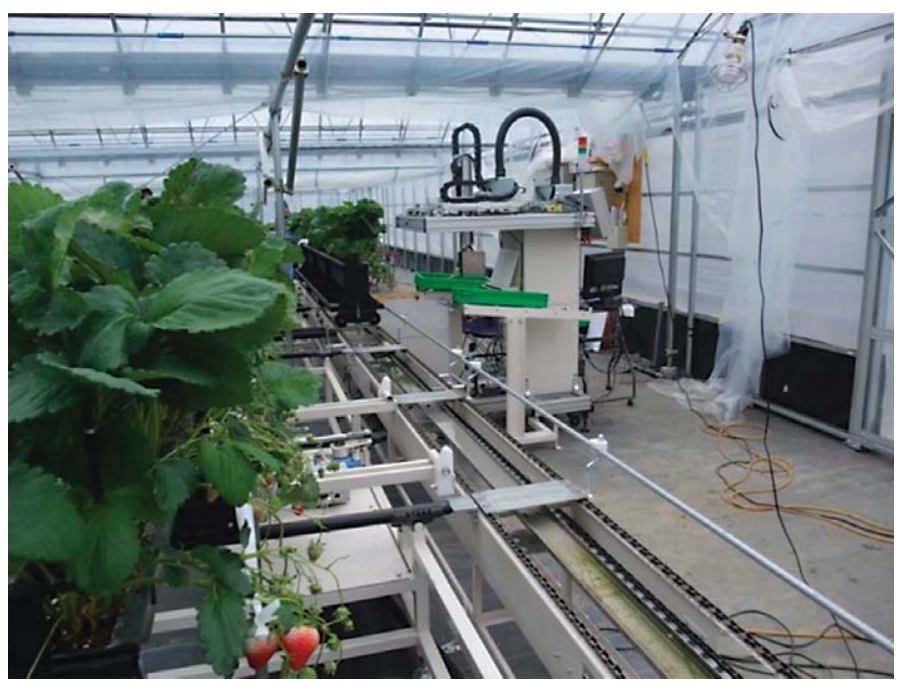

Fig. 7. Stationary-type strawberry-harvesting robot.

sprayer is structured as a gate frame with three spray nozzles (top, left, and right) and is installed in the middle of the lateral conveying unit that is farthest from the workspace.

\subsection{Basic movement of planting bench}

The benches are moved rotationally starting from an initial position. The two benches that are loaded on each lateral conveying unit move simultaneously in the lateral direction and stop at the end. They are then pulled into the longitudinal conveying unit to join the other benches in that unit, so that the farthest benches on the other side are transferred onto the lateral conveyors. After completing one cycle, the benches return to their initial position. This cycle of movement can be repeated, resulting in all of the benches making a complete circuit. The control program has three operational modes: working, watering, and spraying. The speed of lateral conveying can be chosen from among these three speeds according to the type of work.

\subsection{Stationary-type harvesting robot}

A stationary-type harvesting robot, located at the centre of the lateral conveying unit, was developed to be combined with the movable bench system, as shown in Fig. 7. The basic structure and functions are almost the same as those for the movable-type harvesting robot described above, although some parts were improved to make the best use of the stationary mode; that is, two stereo cameras are separated from the end-effector, allowing the machine vision to scan the fruits while the end-effector is placing them into the tray. For daytime operation, shading materials are placed around the robot to block sunlight, and high output LED lights are used.

\subsection{System performance}

The elapsed time during 10 bench movement cycles was recorded using a stopwatch, and the cycle times at three lateral conveying speeds were calculated. The cycle times for each speed mode were $44.5 \mathrm{~s}$ at low speed, $28.8 \mathrm{~s}$ at medium speed, and $23.8 \mathrm{~s}$ at high speed. These results indicate that the waiting time for the next bench arriving in front of the worker can be shortened. Several cultivars are planned to be grown in a forcing culture using the developed system with a plant distance of 12 or $15 \mathrm{~cm}$ on the planting bench, providing the high-density planting of 13-16.7 plants per square meter. 
Functional testing of the stationary-type harvesting robot was carried out, and it was shown that the robot was able to stably perform the picking operation in connection with the movable bench system. It was also verified that the robot can work during the daytime under a structured environment, unless direct sunlight irradiates the target fruits.

\section{Conclusions}

Strawberry production in Japan has several unique features that distinguish it from the cultivation practices in other countries. These include the following. 1) The main crop type is a forcing culture using one-season bearing cultivars. 2) The packing operation is done in a work shed after the picked fruits are pre-cooled in a refrigerator. 3) The fruits are packed by arranging the fruit direction to decrease vibration during transportation, which also makes them visually attractive. These practices lead to long working hours of more than 20000 hours per hectare; the harvesting and packing operations account for approximately one-quarter of the working hours.

We developed a harvesting robot and a packing robot to reduce labour cost. Although the performance accuracy of the harvesting robot still requires improvement, it has reached a level suitable for commercialization. The packing robot was capable of automatically packing strawberries into a single-layer soft tray. Furthermore, the stationary harvesting robot combined with the movable bench system showed great potential to be a new production system for strawberries.

\section{References}

[1] MAFF. Vegetables. The 84th Statistical Yearbook of Ministry of Agriculture. Forestry and Fisheries, Tokyo, Japan, 2007 ; p. 178.

[2] Hancock JF. Tunnels and forcing systems. Strawberries, CABI Publishing, NY, USA, 1999; 122-4.

[3] Harrell RC, Adsit PD, Pool TA, Hoffman R. The Florida robotic grove-lab. Transactions of the ASAE. 1990; 33(2): 391-9.

[4] Plebe A, Grasso G. Localization of spherical fruits for robotic harvesting. Machine Vision and Applications. 2000; 13(2): 70-9.

[5] Sanders KF. Orange harvesting systems review. Biosystems Engineering. 2005; 90(2): 115-25.

[6] De-An Z, Jidong L, Wei J, Ying Z, Yu C. Design and control of an apple harvesting robot. Biosystems Engineering. 2011; 110(2): 112-22.

[7] Kawamura K, Fujiura T, Ura M, Kondo N. Fruit harvesting robot, Journal of the Japanese Society of Agricultural Machinery. 1985; 68(6): 59-67.

[8] Kondo N, Nishitsuji Y, Ling PP, Ting KC. Visual feedback guided robotic cherry tomato harvesting. Transactions of the ASAE. 1996; 39(6): 2331-8.

[9] Hayashi S, Ganno K, Ishii Y, Tanaka I. Robotic harvesting system for eggplants. Japan Agricultural Research Quarterly. 2002; 36(3): 163-8.

[10] Arima S, Kondo N. Cucumber harvesting robot and plant training system. Journal of Robotics and Mechatronics. 1999;11(3):208-12.

[11] Van Henten EJ, Van Tuijl BAJ, Hemming J, Kornet JG, Bontsema J, Van Os EA. Field test of an autonomous cucumber picking robot. Biosystems Engineering. 2003; 86(3): 305-13.

[12] Arima S, Kondo N, Yagi Y, Monta M, Yoshida Y. Harvesting robot for strawberry grown on table top culture (part 1). Journal of Society of High Technology in Agriculture. 2001; 13(3): 159-66.

[13] Cui Y, Nagata M, Gejima Y, Cao Q. Study on strawberry harvesting robot using machine vision for strawberry grown on annual hill top (part 1). Journal of the Japanese Society of Agricultural Machinery. 2006; 68(6): 59-67.

[14] Kondo N, Hisaeda K, Hatou K, Yamashita J, Monta M. Harvesting robot for strawberry grown on annual hill top (part 1). Journal of Society of High Technology in Agriculture. 2000; 12(1): 23-9.

[15] Tillett ND, He H, Tillett RD. Development of a vision guided robot manipulator for packing horticultural produce. Journal of Agricultural Engineering Research. 1995; 61: 145-54.

[16] Ishii T, Toita H, Kondo N, Tahara N. Deciduous fruit grading robot (Part 1). Journal of the Japanese Society of Agricultural Machinery. 2003; 65(6): 163-72.

[17] Kondo N. Machine vision based on optical properties of biomaterials for fruit grading system. Environment Control Biology. 2006; 44(3): 151-9.

[18] Bato PM, Nagata M, Cao Q, Hiyoshi K, Kitahara T. Study on sorting system for strawberry using machine vision (Part 2). Journal of the Japanese Society of Agricultural Machinery. 2000; 62(2): 101-10.

[19] Yamada H, Tanaka N, Takada S. Practical application of a non-destructive analyzer of strawberry quality. Journal of the Illumination Engineering Institute of Japan. 2009; 93(5): 273-7. 
[20] Liming X, Yanchao Z. Automated strawberry grading system based on image processing, Computers and electronics in agriculture. 2010; 71(Sup1): S32-9.

[21] Hayashi S, Shigematsu K, Yamamoto S, Kobayashi K, Kohno Y, Kamata J, Kurita M. Evaluation of a strawberry-harvesting robot in a field test. Biosystems Engineering. 2010; 105: 160-71.

[22] Hayashi S, Yamamoto S, Saito S, Ochiai Y, Nagasaki Y, Kohno Y. Structural environment suited to the operation of a strawberry-harvesting robot mounted on a travelling platform. Engineering in Agricultural Environmental and Food. 2013; 64(1): 34-40.

[23] Yamamoto Y, Ochiai Y, Saito S, Hayashi S. Study on an automatic packing system for strawberries. Applied Engineering in Agriculture. 2012; 28(4): 593-601.

[24] Hayashi S, Saito S, Iwasaki Y, Yamamoto S, Nagoya T, Kano K. Development of circulating-type movable bench system for strawberry cultivation. Japan Agricultural Research Quarterly. 2011a; 45(3): 285-93.

[25] Hayashi S, Takahashi K, Yamamoto S, Saito S, Komeda T. Gentle handling of strawberries using a suction device. Biosystems Engineering. 2011b; 109: 348-56. 